This is a self-archived version of an original article. This version may differ from the original in pagination and typographic details.

Author(s): Lahtinen, Tuomo; Turtiainen, Hannu; Costin, Andrei

Title: BRIMA : Low-Overhead Browser-Only Image Annotation Tool

Year: 2021

Version: Draft (Preprint)

Copyright: @ 2021 IEEE

Rights: In Copyright

Rights url: http://rightsstatements.org/page/InC/1.0/?language=en

Please cite the original version:

Lahtinen, T., Turtiainen, H., \& Costin, A. (2021). BRIMA : Low-Overhead Browser-Only Image Annotation Tool. In ICIP 2021 : Proceedings of the 28th IEEE International Conference on Image Processing (pp. 2633-2637). IEEE. Proceedings : International Conference on Image Processing. https://doi.org/10.1109/icip42928.2021.9506683 


\title{
BRIMA: LOW-OVERHEAD BROWSER-ONLY IMAGE ANNOTATION TOOL (PREPRINT)
}

\author{
Tuomo Lahtinen ${ }^{\dagger}$, Hannu Turtiainen ${ }^{\ddagger}$, Andrei Costin ${ }^{\ddagger *}$ \\ University of Jyväskylä \\ Jyväskylä, Finland \\ $\dagger$ tuomo.t.lahtinen@student.jyu.fi , ${ }^{\ddagger}\{$ ancostin,turthzu $\} @$ jyu.fi
}

Index Terms - Image Annotation, Annotation Tool, Crowdsource Annotation, Image Dataset Generation, COCO

\begin{abstract}
Image annotation and large annotated datasets are crucial parts within the Computer Vision and Artificial Intelligence fields.At the same time, it is well-known and acknowledged by the research community that the image annotation process is challenging, time-consuming and hard to scale. Therefore, the researchers and practitioners are always seeking ways to perform the annotations easier, faster, and at higher quality. Even though several widely used tools exist and the tools' landscape evolved considerably, most of the tools still require intricate technical setups and high levels of technical savviness from its operators and crowdsource contributors.

In order to address such challenges, we develop and present BRIMA - a flexible and open-source browser extension that allows BRowser-only IMage Annotation at considerably lower overheads. Once added to the browser, it instantly allows the user to annotate images easily and efficiently directly from the browser without any installation or setup on the client-side. It also features cross-browser and cross-platform functionality thus presenting itself as a neat tool for researchers within the Computer Vision, Artificial Intelligence, and privacy-related fields.
\end{abstract}

\section{INTRODUCTION}

Research efforts in the fields of Computer Vision, image processing, image annotation, and object detection/recognition often require large datasets of annotated images. In some cases, existing datasets are not suitable or not available, therefore the data must be created fast and from the scratch as part of the research. For image dataset creation purpose, multiple annotation tools have been developed and published during the last decade [1, 2, 3, 4, 5, 6, 7]. In order to create such an object detection model, it requires a large and high-quality dataset of annotated images. Therefore, there is stringent need for fast, easy and scalable image annotation tools with lowest overheads in terms of bootstrapping and usage.

\footnotetext{
*To appear in Proceedings of the 2021 IEEE International Conference on Image Processing (ICIP).
}

This work presents the first browser-only image annotation tool which aids researchers to fast and easy create high-quality image-based datasets for their custom needs. Our browser extension offers a new way to annotate images, where annotation is done directly in the browser (within browser-viewport), does not require additional software setups, and the annotator is in full control which image to annotate (as it comes directly from the browser's viewport), allowing what could be called annotate-while-freely-browsing experience. The support for standard/compatible data formats (e.g., MS COCO [8]) makes our extension compatible with other annotation tools that are used in the computer vision modeling pipelines.

For the browser extension, we set forward a few requirements to ease our collaborative annotation team, in making image annotation with good quality and coverage. Firstly, the extension should be easy and straightforward to use. For example, the user does not need to learn how to use extension more than a few minutes and annotation should be a forward process where the UI of the extension makes user comfortable to perform the annotations at high pace and with high accuracy while offering a good User Experience (UX). Secondly, the extension should require minimal setup. For example, the most recent browsers are known for the ease of installing add-on software, i.e., browser extensions. It is as simple as clicking "Install Add-on" and not even requiring the browser's restart, all that thanks to robust browser architectures and the great flexibility of high-level programming languages such as JavaScript.

Our main contributions with this work are as follow:

- To the best of our knowledge, we develop the first browseronly browser-viewport image annotation toolset

- We validate our tool's effectiveness in a real-world crowdsourcing experiment

- We release the code and artifacts under open-source license

\section{RELATED WORK}

PhotoStuff is an "annotation tool for digital images on the Semantic Web" allowing "to manually annotate images using Web ontologies" proposed by Halaschek et al. [2]. Presumably, it could be run both as a stand-alone as well as a 
web-based application. Wilhelm et al. [9] presented photo annotation with the mobile phone. In their work, some annotation was created automatically and sent to a server for annotation enhancements. LabelMe by Russell et al. [4] is an earlier web-based image annotation tool, that allowed pretty flexible annotation and functionality. Korc and Schneider [7] proposed MATLAB-based Annotation Tool - a stand-alone software employing parts of the LabelMe by Russell et al. [4].

LabelImg [6] is a tool that presents a fast way to perform rectangle annotation to images in PascalVOC [10] and YOLO [11] formats, but it does not support MS COCO [8] JSON format. Labelme by Wada [5] can annotate images with polygon segments that can be subsequently used in object segmentation architectures. It also supports MS COCO [8] JSON format, and includes Python scripts to embed the data as an MS COCO single annotation file format. Labelme by Wada [5] has drawn inspiration from LabelMe by Russell [4]. Ilastik is a stand-alone "easy-to-use interactive tool that brings machine-learning-based (bio) image analysis to end users without substantial computational expertise" developed and proposed by Sommer et al. [12] and Berg et al. [13]. Ciocca et al. [1] developed IAT as a stand-alone annotation tool. Qin et al. [14] propose ByLabel as a new semi-automatic boundary-based image annotation tool, which is also presented as an improved alternative to LabelMe by Russell et al. [4]. Bernal et al. [3] presented GTCreator - a stand-alone "flexible annotation tool for providing image and text annotations to image-based datasets." Finally, for more complete and comprehensive surveys of the major tools and techniques for image annotation, we refer interested readers to Hanbury [15] and Dasiopoulou et al. [16].

Crowdsourcing is a fast and cost-effective way to make image annotation with a potentially large group of collaborators, i.e., annotators. Su et al. [17] presented a system that collects bounding-box (i.e., bbox) annotations through crowdsourcing. Using crowdsourcing, however, creates a significant challenge on how to ensure that annotation quality and coverage is reasonably good [15], [18]. At the same time, crowdsourcing efforts need to pay particular attention to the reliability and differences between annotators during crowdsourcing [19, 17, 18].

\section{DETAILED OVERVIEW OF THE TOOLSET}

The browser extension presented in this work offers a new way to annotate images, and the researchers can create their own dataset that includes images suitable for the exact need.

\subsection{Features}

We have been continuously designing, iterating, and improving the toolset's functionality. At present, BRIMA offers the following attractive and unique combination of features:
- Browser-only. It does not require any installation or setup on the client-side, except of several clicks (e.g., "Install Add-on") when using most common and modern browsers.Our tool allows to capture and annotate whatever is currently displayed in the user browser. Unlike all current tools, this means there is no limitation what images are presented to the annotator. Therefore, it provides more diversity to the final dataset since the annotator can browse whatever URL or image s/he thinks is representative to the annotation task at hand, or according to the crowdsourcing instructions. In a nutshell, with our tool annotation is as simple as annotate-while-freely-browsing.

- Web-oriented. Traditional image annotation tools require that the images are first scraped from the web. Then, most often, they also require images to be organized by folders and filenames. Such an approach increases the complexity, the risk of errors, and the time required to pre-organize the dataset for annotation. In our design, the annotation takes place within the extension and the browser. This means that there is no need to scrape and organize the images to local files and folder, as the web images are already rendered within the extension which at the same time is the actual annotation tool. This also means there is no need to develop and maintain additional code that scrapes the web.

- URL-aware. Our tool by default can store, parse, and otherwise process URLs for various additional data, thanks to being run within the browser. For example, for Google Street View [20] it can parse almost exact location of the annotated object including additional parameters such as zoom and orientation of the Google Street View lens that captured the given view. However, it can be configured and used on virtually any set of websites, according to the needs of the crowdsourcing effort. Also, parsing routines can be added and customized separately on the basis of the domain/subdomain names, as well as prefixes and regular expressions matching certain URL patterns. This also means that there is always a possibility to trace-back where each training/validation/testing image or annotation was taken from. Hence, this allows to further improve the annotation and data quality-check processes.

- Minimal client-side. Our browser extension does not require complex setup and installation on the client-side. Everything can be done in browsers setup where the user just needs to configure/activate the extension. This makes largescale crowdsourcing a breeze.

- Minimal server-side. It also requires minimal server-side setup and configuration. We tested and release minimal setups supporting PHP and Python API backends.

- Supports extensive combinations of "OS + browser". The browser extension can be used in most common browsers and it has been tested with multiple different combinations of OS and browsers (see Section 3.4). 
- Support for standard/compatible data formats. It supports natively MS COCO [8] annotation JSON file formats, thus enabling immediate training of object detectors using a variety of CV/ML framework and backend combinations.

- Out-of-the-box usage. Our toolset can be used immediately and out-of-the-box by taking advantage of the provisioned client-side templates README documentation, and one-line scripts for server-side supporting HTTP/HTTPS with minimal software dependencies.

- Easy to configure. Both the client-side browser extension and the server-side are easy to configure for various real-world scenarios. Most common browsers have a few click solution for activating browser extension and the server-side can be immediately started using a configurable command-line script.

- Easy to use. User interface is simple, and annotation is done with the mouse, with optional support for configurable keyboard shortcut keys. Color-coding helps to identify which object type/category the user is currently annotating (color-coding was noted by Gong et al. [21] as an important feature for annotation).

- Configurable keyboard shortcuts. Multiple keyboard shortcuts are defined, and can be customized. For example, a single key-press to select annotated object type or another key-press to accept all annotated object(s) and to send the full JSON data to the server backend.

- No pre-built dataset required. This is a consequence of the web-oriented design of our toolset, i.e., there is no need for pre-annotation web-scraping phase.

\subsection{Technical details}

Our entire toolset is composed of client-side (i.e., the browser extension), and the server-side which receives and stores the annotated data. The browser extension is written in pure JavaScript, and it is helping the researchers and crowdsource contributors for fast and easy data annotation. For serverside, we provide basic reference implementations in PHP and Python (using Flask and Swagger/OpenAPI). However, nothing limits our toolset's users to rewrite the server-side in a different language and serve it from a different web-server setup. The browser extension and the server communicate with each other via HTTP/XHR and RESTful API requests.

The exact annotation polygon data is based on the input from the user (typically using precise mouse clicks), and other parts of annotation data are automatically generated or enhanced by the browser extension. All the annotation data is sent to the server-side using a JSON format structure which at its core is compatible with the MS COCO and can be immediately used for CV/ML training under various object detection frameworks (e.g., TensorFlow [22]) and backends (e.g., CenterMask2 [23], Detectron2 [24]).

\subsection{Toolset's workflow}

Our browser extension is easy to use. After an initial client/server-side configuration, its usage follows a simple pattern:

1. The user seeks an image from web resources such as photo stock websites (e.g., Flickr) or street view imagery (e.g., Google StreetView) corresponding to dataset collection needs. The key and novel idea is that annotator's browser viewport directly becomes the "image to be annotated".

2. The user uses few keyboard/mouse actions to activate the image into our browser extension, and after that the selected viewport image is ready for annotation.

3 . The user draws the annotation polygon around the object(s) of interest, and confirm object annotation.

4. The user, once all objects of interest are annotated, confirms the annotation is ready, and with a single click sends the data to the data collection server.

5. The server receives the data in MS COCO compatible format, processes it, and is ready to create a complete dataset snapshot ready for CV/ML training.

Figures 1, 2 present our tool in real-life action. In particular, Figure 1 presents our extension used for privacy, antisurveillance and safety applications. In that particular setup we configured it to aid the collection of an annotated CCTV cameras dataset. We use such a dataset to build a state-of-theart object detector for CCTV camera objects in real-life and in street-level imagery [25]. For the case of CCTV camera dataset collection, we easily configured the extension to allow the collection of additional data that is both specific and relevant for CCTV camera object. For example, Figure 1 shows that the annotator can optionally fill the camera's exact model and camera's owner/operator. Alternatively, Figure 2 presents our extension used for other privacy and generic Computer Vision applications. For example, in "other privacy" applications it can be used to collect annotated datasets related to blurred/unblurred persons, faces, and license plate numbers. Also, it can be used for general-purpose CV applications such as collecting accurate annotation of traffic signs and street infrastructure that can be subsequently used by self-driving cars and their autopilots.

\subsection{Toolset's validation}

We validate the effectiveness, efficiency, and usability of BRIMA toolset by actively employing it during a crowdsource effort aimed to create a large dataset used to train the firstever state-of-the-art object detector for CCTV objects [25]. Its purpose is to detect CCTV cameras in various images (e.g., street view) for privacy, anonymity, anti-surveillance, safety applications [26]. Such applications are important due to risks associated with lax cybersecurity of CCTV systems [27], and their "privacy invasion" misuse once hacked. 


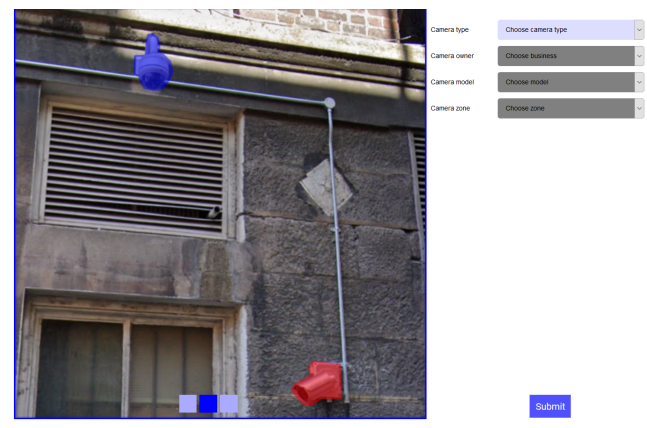

Fig. 1. Browser window when extension is in annotation mode, and the user annotated two CCTV cameras.

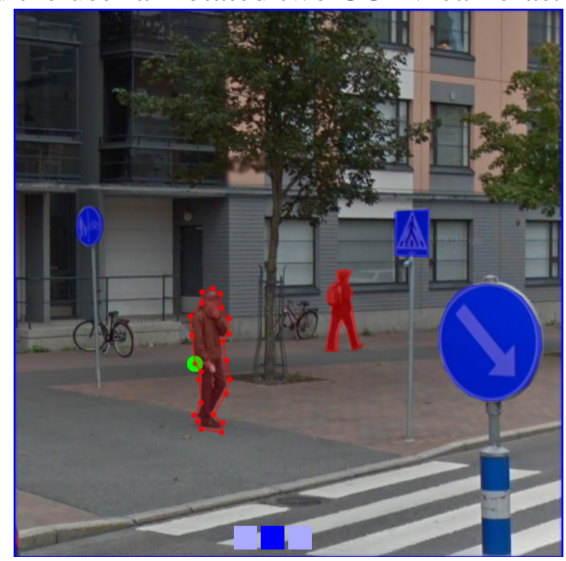

Fig. 2. Browser extension can be used to annotate virtually any object of interest such as faces, humans, traffic signs.

We recruited eight (8) image annotators which are mainly non-experts in crowdsourcing and image annotation. The entire crowdsourcing effort took place in September 2020 and overall, it took an equivalent of at least one and a half personmonth effort. Due to the ease of use and setup, and its weboriented and browser-only design (see Section 3.1), during the 72 hours experiment window the annotators were able to submit 4167 quality-checked images for a total of 5380 annotated instances of CCTV cameras (3325 directed and 2055 round). Dataset results obtained using BRIMA are comparable with the state-of-the-art datasets of annotated images such as MS COCO where 6097 is the median size of a training dataset for the particular object types such as cars, airplanes, stop signs. Thanks to the extension, the work and the crowdsourcing described in this paper, we were able to achieve state-of-the-art results in our wider efforts related to the CCTV camera object detector [25].

Table 1 presents overall summary of the clean and qualitychecked dataset at the end of the crowdsource experiment. In Table 2 we present some detailed statistics for each crowdsourcing annotator and shortly explain each metric below:

- Approved Images. Number of images that successfully passed Quality Check (QC), both in terms of image and annotation.
- DQ. DisQualification rate representing the percentage of images that were disqualified as a result of a failed QC. Hence the original number of submitted images per annotator is usually higher than the approved images.

- Annotation expertise. Self-reporting scale was set from 0 (novice) to 5 (expert).

- Easy setup and use. Self-reporting scale was set from 0 (extremely hard) to 5 (extremely easy).

- Overall experience. Self-reporting scale was set from 0 (extremely bad) to 5 (extremely good).

- OS and browser. The browser extension performed without any issues in a sufficiently diverse set of user environments (5 major OS releases and 3 major browser variants).

Table 1. Crowdsourced dataset statistics.

\begin{tabular}{l|r||l|r}
\hline \multicolumn{1}{c}{ Total counts } & \multicolumn{3}{c}{ Instances grouped by sub-type } \\
\hline Total collected images & 4167 & Directed camera instances & 3325 \\
Total annotated camera instances & 5380 & Round camera instances & 2055 \\
\hline \multicolumn{1}{c}{ Images grouped by source } & \multicolumn{2}{c}{ Instances grouped by area } \\
\hline Google (Street View, Image Search) & 3873 & Small (<32x32 px) & 1455 \\
Baidu street view & 269 & Medium (32x32-96x96 px) & 3345 \\
Flickr & 25 & Large (>96x96 px) & 580 \\
\hline
\end{tabular}

Table 2. Crowdsourcing statistics for each annotator.

\begin{tabular}{|c|c|c|c|c|c|c|}
\hline Annotator & $\begin{array}{c}\text { Approved } \\
\text { Images }\end{array}$ & DQ \% & $\begin{array}{l}\text { OS and browser } \\
\text { (default 64-bit) }\end{array}$ & $\begin{array}{c}\text { Annotation } \\
\text { expertise } \\
(0-5)\end{array}$ & $\begin{array}{c}\text { Easy setup } \\
\text { and use } \\
(0-5)\end{array}$ & $\begin{array}{c}\text { Overall } \\
\text { experience } \\
(0-5)\end{array}$ \\
\hline Person1 JU & 418 & $1.7 \%$ & $\begin{array}{l}\text { MacOS (10.15.6) } \\
\text { FFox (80.0.1) }\end{array}$ & 0 & 3.5 & 2 \\
\hline Person2 PK & 525 & $0.2 \%$ & $\begin{array}{c}\text { Debian (9.13) } \\
\text { Chromium (73.0.3683.75-dev) }\end{array}$ & 0 & 5 & 4 \\
\hline Person3 SK & 542 & $14.4 \%$ & $\begin{array}{c}\text { Win10 } \\
\text { FFox }(81.0 .1)\end{array}$ & 3 & 5 & 4 \\
\hline Person4 AA & 228 & $15.4 \%$ & no response & no response & no response & no response \\
\hline Person5 AT & 632 & $3.0 \%$ & $\begin{array}{c}\text { Win10 (1809) } \\
\text { FFox (78.3.0esr) }\end{array}$ & 1 & 3 & 4 \\
\hline Person6 TL & 750 & $0.7 \%$ & $\begin{array}{c}\text { Win10 } \\
\text { FFox }(80.0 .1)\end{array}$ & 2 & 5 & 4 \\
\hline Person7 LS & 977 & $5.4 \%$ & $\begin{array}{c}\text { Ubuntu (18.04.5 LTS) } \\
\text { Chrome (85.0.4183.121) }\end{array}$ & 1 & 5 & 5 \\
\hline Person8 AC & 95 & $0.0 \%$ & $\begin{array}{c}\text { Ubuntu (16.04 LTS) } \\
\text { FFox (68.7.0esr) 32-bit }\end{array}$ & 4 & 5 & 5 \\
\hline$\frac{\text { avg. }}{\text { sum }}$ & $\frac{520.87}{4167}$ & $\frac{4.75 \%}{-}$ & - & $\frac{1.57}{-}$ & $\frac{4.5}{-}$ & $\underline{4}$ \\
\hline
\end{tabular}

\section{CONCLUSION}

We presented BRIMA - the first browser-only annotation toolset that allows easy and fast image annotation within browser-viewport while incurring a low overhead overall. We also validated the effectiveness, efficiency, and usability of our extension/toolset by actively employing it during a successful crowdsource effort. This underpins the fast-tobootstrap and easy-to-maintain characteristics of our toolset and the extension, therefore allowing the researchers to actually focus on the core of their experiments (e.g., training and experimenting state-of-the-art object detectors for various applications). We believe our toolset is a valuable contribution to the communities of researchers and practitioners, therefore the relevant artefacts (code, documentation, samples) will be made available open-source at: https://github.com/tutalaht/brima 


\section{Acknowledgment}

Authors would like to acknowledge grants of computer capacity from the Finnish Grid and Cloud Infrastructure (FGCI) (persistent identifier urn:nbn:fi:research-infras-2016072533).

Part of this research was kindly supported by the "Decision of the Research Dean on research funding within the Faculty (17.06.2020)" grant from the Faculty of Information Technology of University of Jyväskylä (the grant was facilitated and managed by Dr. Andrei Costin).

Hannu Turtiainen would like to also thank:

- The Finnish Cultural Foundation / Suomen Kulttuurirahasto (https://skr.fi/en) for supporting his Ph.D. dissertation work and research (grant decision 00211119).

- The Faculty of Information Technology of University of Jyvaskyla (JYU), in particular Prof. Timo Hämäläinen, for partly supporting his Ph.D. supervission at JYU in 2021.

\section{REFERENCES}

[1] Gianluigi Ciocca, Paolo Napoletano, and Raimondo Schettini, "IAT Image Annotation Tool: Manual," preprint arXiv:1502.05212, 2015.

[2] WC Halaschek, J Golbeck, A Schain, M Grove, B Parsia, and J Hendler, "PhotoStuff: An image annotation tool for the semantic web," in Proceedings of the Poster Track, 4th International Semantic Web Conference, 2005, pp. 2-4.

[3] Jorge Bernal et al., "GTCreator: a flexible annotation tool for imagebased datasets," International journal of computer assisted radiology and surgery, vol. 14, no. 2, pp. 191-201, 2019.

[4] BryanC. Russell, Antonio Torralba, KevinP. Murphy, and WilliamT. Freeman, "LabelMe: A Database and Web-Based Tool for Image Annotation," International Journal of Computer Vision, vol. 77, no. 1-3, pp. 157-173, 2008.

[5] Kentaro Wada, "labelme: Image Polygonal Annotation with Python," 2016.

[6] Tzutalin, "LabelImg - a graphical image annotation tool and label object bounding boxes in images," 2015

[7] Filip Korc and David Schneider, "Annotation tool," Dept. Photogrammetry, Univ. Bonn, Bonn, Switzerland, TR-IGG-P-2007-01, 2007.

[8] Tsung-Yi Lin, Michael Maire, Serge Belongie, James Hays, Pietro Perona, Deva Ramanan, Piotr Dollár, and C Lawrence Zitnick, "Microsoft COCO: Common objects in context," in European Conference on Computer Vision. Springer, 2014.

[9] Anita Wilhelm, Yuri Takhteyev, Risto Sarvas, Nancy Van House, and Marc Davis, "Photo annotation on a camera phone," in CHI'04 extended abstracts on Human factors in computing systems, 2004.

[10] Mark Everingham, Luc Van Gool, C. K. I. Williams, J. Winn, and Andrew Zisserman, "The PASCAL Visual Object Classes (VOC) challenge," 2010.

[11] Joseph Redmon and Ali Farhadi, "YOLOv3: An Incremental Improvement," 2018.

[12] Christoph Sommer, Christoph Straehle, Ullrich Koethe, and Fred A Hamprecht, "Ilastik: Interactive learning and segmentation toolkit," in 2011 IEEE international symposium on biomedical imaging: From nano to macro. IEEE, 2011, pp. 230-233.

[13] Stuart Berg, Dominik Kutra, Thorben Kroeger, Christoph N Straehle, Bernhard X Kausler, Carsten Haubold, Martin Schiegg, Janez Ales, Thorsten Beier, Markus Rudy, et al., "Ilastik: interactive machine learning for (bio) image analysis," Nature Methods, pp. 1-7, 2019.

[14] Xuebin Qin, Shida He, Zichen Zhang, Masood Dehghan, and Martin Jagersand, "Bylabel: A boundary based semi-automatic image annotation tool," in 2018 IEEE Winter Conference on Applications of Computer Vision (WACV). IEEE, 2018, pp. 1804-1813.
[15] Allan Hanbury, "A survey of methods for image annotation," Journal of Visual Languages \& Computing, vol. 19, no. 5, pp. 617-627, 2008.

[16] Stamatia Dasiopoulou, Eirini Giannakidou, Georgios Litos, Polyxeni Malasioti, and Yiannis Kompatsiaris, "A survey of semantic image and video annotation tools," in Knowledge-driven multimedia information extraction and ontology evolution, pp. 196-239. Springer, 2011.

[17] Hao Su, Jia Deng, and Li Fei-Fei, "Crowdsourcing annotations for visual object detection," in Workshops at the Twenty-Sixth AAAI Conference on Artificial Intelligence, 2012.

[18] Peter Welinder and Pietro Perona, "Online crowdsourcing: rating annotators and obtaining cost-effective labels," in Conference on Computer Vision and Pattern Recognition-Workshops. IEEE, 2010.

[19] Stefanie Nowak and Stefan Rüger, "How reliable are annotations via crowdsourcing: a study about inter-annotator agreement for multi-label image annotation," in Proceedings of the international conference on Multimedia information retrieval, 2010, pp. 557-566.

[20] Dragomir Anguelov, Carole Dulong, Daniel Filip, Christian Frueh, Stéphane Lafon, Richard Lyon, Abhijit Ogale, Luc Vincent, and Josh Weaver, "Google street view: Capturing the world at street level," Computer, vol. 43, no. 6, pp. 32-38, 2010.

[21] Yihong Gong, Hongjiang Zhang, et al., "An image database system with content capturing and fast image indexing abilities," in 1994 Proceedings of IEEE international conference on multimedia computing and systems. IEEE, 1994, pp. 121-130.

[22] Martin Abadi et al., "Tensorflow: Large-scale machine learning on heterogeneous distributed systems," 2016.

[23] Youngwan Lee and Jongyoul Park, "CenterMask2: Real-Time AnchorFree Instance Segmentation,” 2020.

[24] Yuxin Wu, Alexander Kirillov, Francisco Massa, Wan-Yen Lo, and Ross Girshick, "Detectron2: A PyTorch-based modular object detection library,"

[25] Hannu Turtiainen, Andrei Costin, Tuomo Lahtinen, Lauri Sintonen, and Timo Hamalainen, "Towards large-scale, automated, accurate detection of CCTV camera objects using computer vision. Applications and implications for privacy, safety, and cybersecurity.(Preprint)," arXiv preprint arXiv:2006.03870, 2020.

[26] Tuomo Lahtinen, Lauri Sintonen, Hannu Turtiainen, and Andrei Costin, "Towards CCTV-aware Routing and Navigation for Privacy, Anonymity, and Safety - Feasibility Study in Jyväskylä," in Proceedings of Conference of Open Innovations Association FRUCT, 2021.

[27] Andrei Costin, "Security of CCTV and video surveillance systems: Threats, vulnerabilities, attacks, and mitigations," in 6th International Workshop on Trustworthy Embedded Devices (TrustED), 2016. 